\title{
DNA-binding residues and binding mode prediction with binding-mechanism concerned models

\author{
Yu-Feng Huang ${ }^{1}$, Chun-Chin Huang ${ }^{2}$, Yu-Cheng Liu ${ }^{3}$, Yen-Jen Oyang ${ }^{1,4,5}$
} and Chien-Kang Huang*2
}

\begin{abstract}
Addresses: ${ }^{1}$ Department of Computer Science and Information Engineering, National Taiwan University, Taipei, 106, Taiwan, Republic of China, ${ }^{2}$ Department of Engineering Science and Ocean Engineering, National Taiwan University, Taipei, 106, Taiwan, Republic of China, ${ }^{3}$ Institute of Biomedical Engineering, National Taiwan University, Taipei, 106, Taiwan, Republic of China, ${ }^{4}$ Graduate Institute of Biomedical Electronics and Bioinformatics, National Taiwan University, Taipei, 106, Taiwan, Republic of China and ${ }^{5}$ Center for Systems Biology and Bioinformatics, National Taiwan University, Taipei, 106, Taiwan, Republic of China

E-mail: Yu-Feng Huang - yfhuang@csie.ntu.edu.tw; Chun-Chin Huang - r96525072@ntu.edu.tw; Yu-Cheng Liu - F90548051@ntu.edu.tw; Yen-Jen Oyang - yjoyang@csie.ntu.edu.tw; Chien-Kang Huang* - ckhuang@ntu.edu.tw

*Corresponding author
\end{abstract}

from Asia Pacific Bioinformatics Network (APBioNet) Eighth International Conference on Bioinformatics (InCoB2009)

Singapore 7-II September 2009

Published: 3 December 2009

BMC Genomics 2009, I0(Suppl 3):S23 doi: 10.1186/1471-2164-10-S3-S23

This article is available from: http://www.biomedcentral.com/I47I-2/64/I0/S3/S23

(c) 2009 Huang et al; licensee BioMed Central Ltd.

This is an open access article distributed under the terms of the Creative Commons Attribution License (http://creativecommons.org/licenses/by/2.0), which permits unrestricted use, distribution, and reproduction in any medium, provided the original work is properly cited.

\begin{abstract}
Background: Protein-DNA interactions are essential for fundamental biological activities including DNA transcription, replication, packaging, repair and rearrangement. Proteins interacting with DNA can be classified into two categories of binding mechanisms - sequence-specific and nonspecific binding. Protein-DNA specific binding provides a mechanism to recognize correct nucleotide base pairs for sequence-specific identification. Protein-DNA non-specific binding shows sequence independent interaction for accelerated targeting by interacting with DNA backbone. Both sequence-specific and non-specific binding residues contribute to their roles for interaction.

Results: The proposed framework has two stage predictors: DNA-binding residues prediction and binding mode prediction. In the first stage - DNA-binding residues prediction, the predictor for DNA specific binding residues achieves $96.45 \%$ accuracy with $50.14 \%$ sensitivity, $99.31 \%$ specificity, $81.70 \%$ precision, and $62.15 \%$ F-measure. The predictor for DNA non-specific binding residues achieves $89.14 \%$ accuracy with $53.06 \%$ sensitivity, $95.25 \%$ specificity, $65.47 \%$ precision, and $58.62 \%$ F-measure. While combining prediction results of sequence-specific and non-specific binding residues with OR operation, the predictor achieves $89.26 \%$ accuracy with $56.86 \%$ sensitivity, $95.63 \%$ specificity, $71.92 \%$ precision, and $63.51 \% \mathrm{~F}$-measure. In the second stage, protein-DNA binding mode prediction achieves $75.83 \%$ accuracy while using support vector machine with multi-class prediction.

Conclusion: This article presents the design of a sequence based predictor aiming to identify sequence-specific and non-specific binding residues in a transcription factor with DNA bindingmechanism concerned. The protein-DNA binding mode prediction was introduced to help improve DNA-binding residues prediction. In addition, the results of this study will help with the design of binding-mechanism concerned predictors for other families of proteins interacting with DNA.
\end{abstract}




\section{Background}

Protein-DNA interactions play important roles for the regulation of key biological functions like DNA transcription, replication, packaging and recombination. With the increasing number of high quality structure of complexes in Protein Data Bank (PDB) [1] and Nucleic Acid Database (NDB) [2], the collection of atomic interaction information for their structural and functional integrity is sufficiently complete for analysis and prediction of protein-nucleic acid interactions. Atomic level analyses have been investigated to understand how amino acids interact with nucleotide bases or sugarphosphate backbones through hydrogen bonds, van der Waals contacts, or water-mediated hydrogen bonds [3], depending on the amino acid propensities [4,5]. In recent years, the prediction of residues in a protein chain that interact with DNA has been a research topic that attracts a high level of interest. Some of the studies were purely based on analysis of the protein polypeptide sequence [6-11], while the others took the structural information into account [12-17]. Particularly, the issue for sequence-specific binding residue prediction has been also mentioned recently [18]. Transcription factors (TFs) are proteins that regulate gene expression, which serve as integration centers of the different signaltransduction pathways affecting a given gene [19]. TFs regulate cell development, differentiation, and cell growth by binding to a specific DNA site and regulating gene expression [20-22]. As it has been reported in a recent article that the tertiary structures of a large number of TFs are mostly disordered [23], sequence based analysis aimed at identifying the residues in a highlydisordered TF that play key roles in interaction with the DNA is essential for obtaining a comprehensive picture of how TFs function.

As studied in previous research, proteins that interact with DNA will change their conformations from their free states, changing non-specific complexes to specific complexes [24]. During the course of DNA-recognition, residues play different roles to either recognize nucleotide bases or stabilize the protein-DNA conformation. In this work, we try to identify whether the residue performs sequence-specific or non-specific binding. There are two types of binding mechanisms involved in amino acid - nucleotide interactions, namely sequencespecific and non-specific site binding [25-29]. Sequencespecific binding occurs between protein side-chains and nucleotide bases, while non-specific binding occurs between protein side-chains and the DNA sugar/phosphate backbone [28]. In general, sequence-specific binding is also named as specific binding. Specific binding corresponds to sequence-specific recognition of a gene and therefore is essential for the correct regulation of genes. Non-specific binding shows relatively little base-sequence preference and binds preferentially to either single or double-stranded DNA. The role for nonspecific binding residues is to stabilize the interactions between protein and nucleotide backbone to help specific binding residues in recognizing base pairs correctly. As reported in the review article by Luscombe et al. [30], protein-DNA interactions can be grouped into eight different structural/functional groups based on the structures of the DNA-binding region in the proteins, which is also referred to as the binding mode of the protein [30-32]. There are eight such binding modes including (I) Helix-Turn-Helix, HTH (including "winged" HTH), (II) zinc-coordinating, (III) zippertype, (IV) other $\alpha$-helix, (V) $\beta$-sheet, (VI) $\beta$-hairpin/ ribbon, (VII) other, (VIII) enzymes. Related research has investigated the classification of protein-DNA complexes and structural domains [33-35]. Proteins in the same class have similar binding site conformations despite having different DNA targets. The importance of introducing the DNA-binding mode information is to find the binding pattern that a protein uses to interact with the target DNA [36,37], which could help to identify the location of sequence-specific and nonspecific binding residues.

This article presents the design of a sequence based predictor for identifying the residues in a TF that are involved in both sequence-specific binding and nonspecific binding with the DNA and the binding mode. We use support vector machine (SVM) as the classifier to predict binding residues as sequence-specific or nonspecific according to binding specificity. Originally, the definition of sequence-specific binding and the nonspecific binding residues is based on the identification of hydrogen bonds and van der Waals attractions between protein side-chains and DNAs. In this work, we use a computational approximation of distance cut-off to define binding classification instead. A residue is regarded as involved in sequence-specific binding with the DNA if one or more heavy atoms on its side-chain are within $4.5 \AA$ from any of the nucleic bases, while a residue is regarded as involved in non-specific binding with the DNA if one or more heavy atoms on its sidechain are within $4.5 \AA$ from the sugar/phosphate backbone of the DNA. The threshold of distance cut-off is based on hydrogen bonding and van der Waals attractions: (1) a hydrogen bond was defined as having a maximum donor acceptor distance of $3.35 \AA$ and maximum hydrogen-acceptor distance of $2.7 \AA$. (2) atoms were considered to form van der Waals contacts if the distance between them was $\leq 3.9 \AA$ and the contact had not been defined as a hydrogen bond [5]. Residues in a protein interacting with DNA play their roles on specific binding, or non-specific binding, or both. The reason to predict both sequence-specific and non-specific 
binding residues is that the main determinants of specificity are the unfavorable contributions of "wrong" base pairs and specific binding will also require a large non-specific contribution to the binding free energy to achieve sufficient binding affinity [38]. Furthermore, the information of the predicted sequence-binding and nonspecific binding residues can be used protein-DNA binding mode prediction. As shown in Figure 1, this is an example of PDB ID 2PRT:A to show sequence-specific and non-specific binding residues in the tertiary structure. Residues colored by red for sequence-specific binding residues, blue for non-specific binding residues, and purple for both sequence-specific and non-specific binding residues.

\section{Results and discussion}

In this section, we will report the experiments conducted to evaluate the performance of our proposed approach. In the experiments of the first stage, we repeated the same testing procedure 20 times with randomly and independently generated testing data sets. The independent testing data set used in each run was derived from $30 \mathrm{TF}$ chains randomly selected from the 253 TF-DNA complexes that we have collected (see Materials and Methods for details). In order to eliminate possible bias present in our collection of TF complexes, we took steps

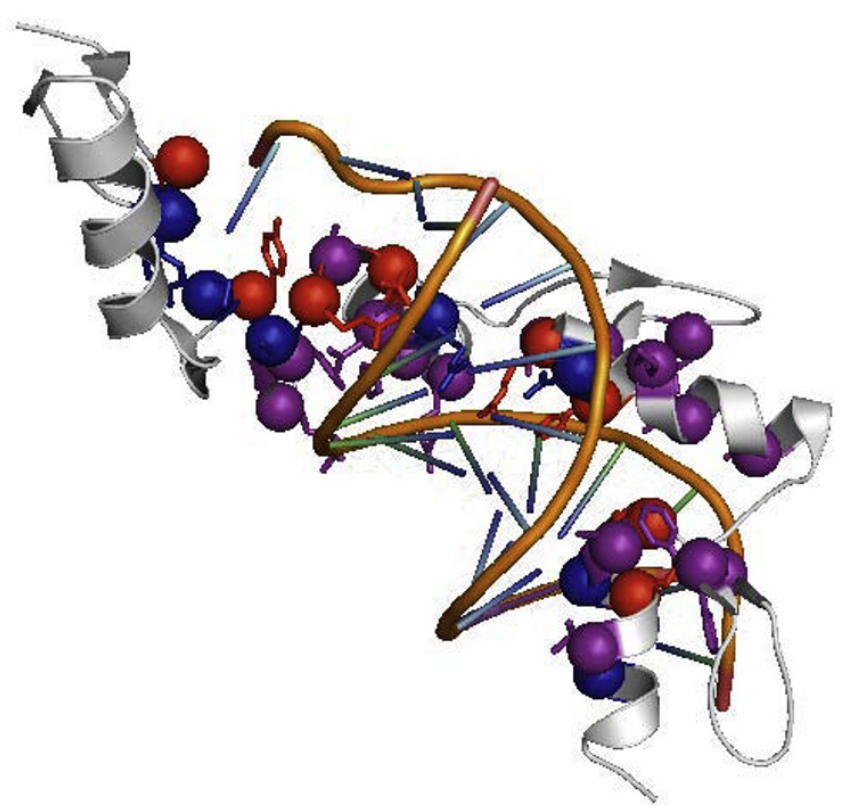

Figure I

Sequence-specific and non-specific binding residues of PDB 2PRT:A. Residues colored by red are sequencespecific binding residues. Residues colored by blue are nonspecific binding residues. Residues colored by purple are both sequence-specific and non-specific binding residues. to guarantee that no two TF chains used to generate the testing data set in the same run are homologous with a sequence identity higher than $20 \%$. Furthermore, aiming to obtain experimental results that accurately reflect the actual performance observed by the users of our proposed approach, we guaranteed that the training data generated with a TF chain that is homologous to the protein chain under testing by having a sequence identity higher than $20 \%$ are removed. For this study, LIBSVM http://www.csie.ntu.edu.tw/ cjlin/libsvm was used for data training and classification [39]. Table 1 shows the overall performance of the SVM predictor for predicting sequence-specific and non-specific binding residues in the first stage. The results have been obtained using the training parameters, $\mathrm{C}=2^{2}, \gamma=2^{-5}$, class weight for binding residue is 1.5 , and class weight for nonbinding residue is 1 , which give better results than other values for prediction of sequence-specific binding residues. The predictor for DNA specific binding residues achieves $96.45 \%$ accuracy with $50.14 \%$ sensitivity, $99.31 \%$ specificity, $81.70 \%$ precision, and $62.15 \%$ F-measure. The results have been obtained using the training parameters, $\mathrm{C}=2^{0}, \gamma=2^{-5}$, class weight for binding residue is 2 , and class weight for non-binding residue is 1 , which give better results than other values for prediction of non-specific binding residues. The predictor for DNA non-specific binding residues achieves $89.14 \%$ accuracy with $53.06 \%$ sensitivity, $95.25 \%$ specificity, $65.47 \%$ precision, and $58.62 \%$ F-measure. While combining prediction results of sequence-specific and non-specific binding residues with OR operation, the predictor achieves $89.26 \%$ accuracy with $56.86 \%$ sensitivity, $95.63 \%$ specificity, $71.92 \%$ precision, and $63.51 \%$ F-measure. Table 2 shows the breakdown of overall performance of the binding residues prediction in terms of secondary structure elements. The number of sequence-specific (or non-specific) binding residues in $\beta$ sheet secondary structure elements is far fewer than the number of sequence-specific (or non-specific) binding residues in either $\alpha$-helix or coil elements. As a result, our proposed framework cannot learn sufficient clues in order to identify sequence-specific (or non-specific) binding residues in $\beta$-sheet elements.

In the experiments of the second stage, the protein-DNA binding mode prediction achieves $75.83 \%$ overall accuracy while applying LIBSVM with multi-class prediction using one-against-one approach. As shown in Table 3, the predictor can deliver precision of $100 \%$ and sensitivity of $80.22 \%$ for zipper-type binding mode, precision of $70.45 \%$ and sensitivity of $73.46 \%$ for helixturn-helix binding mode, precision of $68.07 \%$ and sensitivity of $88.98 \%$ for zinc-coordinating binding mode, and precision of $34.21 \%$ and sensitivity of $52.00 \%$ for $\beta$-hairpin/ribbon binding mode. The 
Table I: Overall performance of proposed approach

\begin{tabular}{|c|c|c|c|c|c|c|c|c|c|}
\hline Binding type & \# of residues & TP & FP & TN & $\mathbf{F N}$ & Sensitivity & Specificity & Precision & Accuracy \\
\hline Sequence-specific binding & 60466 & 1764 & 395 & 56553 & 1754 & $50.14 \%$ & $99.31 \%$ & $81.70 \%$ & $96.45 \%$ \\
\hline Non-specific binding & 60466 & 4652 & 2454 & 49245 & 4115 & $53.06 \%$ & $95.25 \%$ & $65.47 \%$ & $89.14 \%$ \\
\hline Specific+Non-specific & 60466 & $565 I$ & 2206 & 48321 & 4288 & $56.86 \%$ & $95.63 \%$ & $71.92 \%$ & $89.26 \%$ \\
\hline
\end{tabular}

Table 2: Performance of the binding site prediction in terms of secondary structure elements

\begin{tabular}{|c|c|c|c|c|c|c|c|c|c|c|}
\hline Binding type & $\begin{array}{c}\text { Secondary structure } \\
\text { elements }\end{array}$ & $\begin{array}{c}\text { \# of } \\
\text { residues }\end{array}$ & TP & $\mathbf{F P}$ & TN & $\mathbf{F P}$ & Sensitivity & Specificity & Precision & Accuracy \\
\hline Specific & $\begin{array}{c}\text { Helix } \\
\text { Sheet } \\
\text { Coil }\end{array}$ & $\begin{array}{c}32670 \\
5259 \\
22537\end{array}$ & $\begin{array}{c}1322 \\
22 \\
420\end{array}$ & $\begin{array}{c}279 \\
0 \\
116\end{array}$ & $\begin{array}{c}30160 \\
5077 \\
21316\end{array}$ & $\begin{array}{l}909 \\
160 \\
685\end{array}$ & $\begin{array}{l}59.26 \% \\
12.09 \% \\
38.01 \%\end{array}$ & $\begin{array}{c}99.08 \% \\
100.00 \% \\
99.46 \%\end{array}$ & $\begin{array}{c}82.57 \% \\
100.00 \% \\
78.36 \%\end{array}$ & $\begin{array}{l}96.36 \% \\
96.96 \% \\
96.45 \%\end{array}$ \\
\hline Non-specific & $\begin{array}{l}\text { Helix } \\
\text { Sheet } \\
\text { Coil }\end{array}$ & $\begin{array}{c}32670 \\
5259 \\
22537\end{array}$ & $\begin{array}{l}2197 \\
257 \\
2198\end{array}$ & $\begin{array}{l}1005 \\
185 \\
1264\end{array}$ & $\begin{array}{c}27458 \\
4524 \\
17263\end{array}$ & $\begin{array}{c}2010 \\
293 \\
1812\end{array}$ & $\begin{array}{l}52.22 \% \\
46.73 \% \\
54.81 \%\end{array}$ & $\begin{array}{l}96.47 \% \\
96.07 \% \\
93.18 \%\end{array}$ & $\begin{array}{l}66.61 \% \\
58.15 \% \\
63.49 \%\end{array}$ & $\begin{array}{l}90.77 \% \\
90.91 \% \\
86.35 \%\end{array}$ \\
\hline Specific + Non-specific & $\begin{array}{l}\text { Helix } \\
\text { Sheet } \\
\text { Coil }\end{array}$ & $\begin{array}{c}32670 \\
5259 \\
22537\end{array}$ & $\begin{array}{l}2988 \\
261 \\
2402\end{array}$ & $\begin{array}{l}858 \\
181 \\
1167\end{array}$ & $\begin{array}{c}26783 \\
4472 \\
17066\end{array}$ & $\begin{array}{c}2041 \\
345 \\
1902\end{array}$ & $\begin{array}{l}59.42 \% \\
43.07 \% \\
55 / 81 \%\end{array}$ & $\begin{array}{l}96.90 \% \\
96.11 \% \\
93.60 \%\end{array}$ & $\begin{array}{l}77.69 \% \\
59.05 \% \\
67.305\end{array}$ & $\begin{array}{l}91.13 \% \\
90.00 \% \\
86.38 \%\end{array}$ \\
\hline
\end{tabular}

Table 3: Overall performance of protein-DNA binding mode prediction

\begin{tabular}{lccc}
\hline $\begin{array}{l}\text { Protein-DNA binding } \\
\text { mode }\end{array}$ & $\begin{array}{c}\text { \# of protein } \\
\text { chains }\end{array}$ & Sensitivity & Precision \\
\hline zipper-type & 146 & $100.00 \%$ & $80.22 \%$ \\
helix-turn-helix (HTH) & 220 & $70.45 \%$ & $73.46 \%$ \\
zinc-coordinating & 166 & $68.07 \%$ & $88.98 \%$ \\
$\beta$-hairpin/ribbon & 38 & $34.21 \%$ & $52.00 \%$ \\
others & 30 & $93.33 \%$ & $50.91 \%$ \\
\hline
\end{tabular}

predictor did not perform well for TFs with a binding mode of $\beta$-hairpin/ribbon. The reason is that the prediction power of sequence-specific binding and non-specific binding residue on $\beta$-sheet structure is worse than that of $\alpha$-helix and coil. We select PDB 1LMB:4 as an example to show how the predicted binding mode information can be used to enhance the binding residues prediction. Figure 2 displays the prediction result of PDB ID 1LMB:4, which is a difficult case in our binding residues prediction experiment. The protein, 1LMB:4, belongs to the HTH_3 domain which is classified in the group of helix-turn-helix, which has 10 sequence-specific binding residues and 18 non-specific binding residues. However, the predictor found no sequence-specific binding residues with 10 false negatives and found 4 non-specific binding residues with 14 false negatives and 5 false positives. The binding mode predictor can correctly classify the 1LMB:4 into helixturn-helix group. According to the best alignments of secondary structure elements, a protein is selected from the helix-turn-helix group. In Figure 2, residues are colored by red for false positive, blue for false negative and green for true positive. Figure 2(a), 2(b), 2(c) show the prediction of sequence-specific binding residues, the prediction of non-specific binding residues, and the combined result, respectively. Figure 2(d) shows the enhanced prediction with the best aligned template of correct protein-DNA binding mode prediction. It is obviously that correct binding mode prediction can greatly help the binding residues prediction, especially in difficult case. However, this idea needs more investment to derive a systematic approach.

In the following section, we will discuss how the proposed approach performs in comparison with the related studies reported in recent years. One must note that our proposed approach is the only predictor listed in Table 4 that identifies the residues involved in both sequence-specific and non-specific binding with DNA, while all the other predictors do not distinguish between sequence-specific binding and non-specific binding. Since the results listed in Table 4 include the main results extracted from recent studies along with the overall results with our proposed approach, it should be regarded as a survey of the latest advances in the field. It must also be noted that most related studies have adopted slightly different definitions of DNA-binding residues. In the article by Ahmad and Sarai [10] and in the article by Wang and Brown [40], a residue is regarded as involved in interaction with the DNA if one of its heavy atom is within $3.5 \AA$ from a heavy atom of the DNA. In the article by Hwang et al., a larger threshold of $4.5 \AA$ is used instead of $3.5 \AA$. In the article by Yan et al. [8], a residue is regarded as involved in interaction with the DNA if its solvent accessible surface area (ASA) in the 


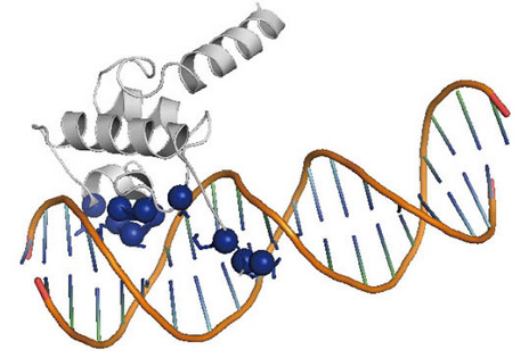

(a) Prediction of sequence-specific binding residues.

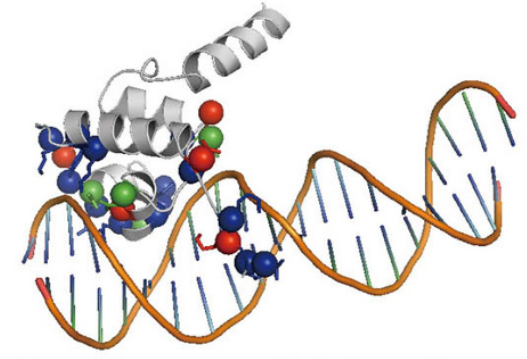

(b) Prediction of non-specific binding residues.



(c) Combination of sequence-specific and non-specific binding residues prediction.

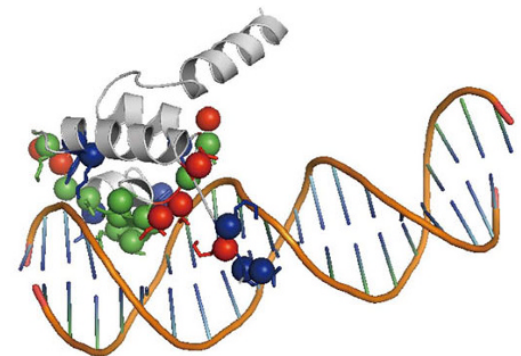

(d) Enhanced prediction with the best aligned template of correct protein-DNA binding mode prediction.

Figure 2

A difficult case (PDB ID ILMB:4) of binding residue prediction, which can be enhanced with the best aligned template of correct predicted protein-DNA binding mode. Residues colored by red means false positive. Residues colored by blue means false negative. Residues colored by green means true positive.

(a) Prediction of sequence-specific binding residues.

(b) Prediction of non-specific binding residues.

(c) Combination of sequence-specific and non-specific binding residues prediction. (d) Enhanced prediction with the best aligned template of correct protein-DNA binding mode prediction.
protein-DNA complex is less than its ASA in the unbound protein by more than $1 \AA^{2}$.

The numbers listed in Table 4 with an asterisk have been derived from the numbers reported in the related studies. Since all the four related studies addressed in Table 4 reported three out of the four performance metrics listed in the table, we can obtain 3 equations about the following 4 variables for each of the related study:

$$
\begin{aligned}
& \widehat{\mathrm{TP}}=\frac{\mathrm{TP}}{\mathrm{TP}+\mathrm{FP}+\mathrm{TN}+\mathrm{FN}}, \widehat{\mathrm{FP}}=\frac{\mathrm{FP}}{\mathrm{TP}+\mathrm{FP}+\mathrm{TN}+\mathrm{FN}}, \\
& \widehat{\mathrm{TN}}=\frac{\mathrm{TN}}{\mathrm{TP}+\mathrm{FP}+\mathrm{TN}+\mathrm{FN}}, \widehat{\mathrm{FN}}=\frac{\mathrm{FN}}{\mathrm{TP}+\mathrm{FP}+\mathrm{TN}+\mathrm{FN}} .
\end{aligned}
$$

In addition, we have $\widehat{\mathrm{TP}}+\widehat{\mathrm{FP}}+\widehat{\mathrm{TN}}+\widehat{\mathrm{FN}}=1$. Therefore, for each related study, we can derive the actual value of the fourth performance metric based on the values of the other three performance metrics that were provided. The only exception is precision for the predictor proposed by Hwang et al. [7]. By definition, the accuracy cannot be higher than the sensitivity and the specificity simultaneously, which is the case with the numbers reported by Hwang et al. Therefore, there is no way to derive the exact value of precision for their predictor.

According to the observation of the predicted results, the predictor of non-specific binding residues tries to locate positive charged patches. However, not all positive charged patches in a protein will come into contact with single- or double-strand DNA. It might be the reason of the performance gap between sequencespecific and non-specific binding residue prediction. While combining prediction results of sequence-specific and non-specific binding residues, sensitivity is higher than other predictors. The reason is that non-specific binding residues help a protein to slide along the target DNA, and specific binding residues will recognize base pairs while sliding along the target DNA. The role the non-specific binding residues play is to help specific binding residues recognize base pairs precisely. Therefore, the prediction of non-specific binding residues can increase the predictor's capability for predicting DNAbinding residues.

\section{Conclusion}

This article presents the design of a sequence based predictor that aims to identify the sequence-specific and non-specific DNA-binding residues in a TF. As a recent study has revealed that the tertiary structures of a large number of transcription factors are mostly disordered, a sequence based predictor is essential for analyzing how a TF interacts with DNA. Furthermore, it is highly desirable to have a predictor capable of identifying the residues involved in sequence-specific binding with DNA, since 
Table 4: Performance delivered by alternative predictors of DNA-binding residues, where the F-measure is the harmonic mean of precision and sensitivity

\begin{tabular}{|c|c|c|c|c|c|}
\hline Predictor & Sensitivity & Specificity & Accuracy & Precision & F-measure \\
\hline Sequence-specific binding & 0.501 & 0.993 & 0.965 & 0.817 & 0.622 \\
\hline Non-specific binding & 0.530 & 0.953 & 0.891 & 0.655 & 0.586 \\
\hline Specific+Non-specific & 0.569 & 0.956 & 0.893 & 0.719 & 0.635 \\
\hline Ahmad and Sarai $[10]$ & 0.682 & 0.660 & 0.664 & $0.308^{*}$ & $0.425^{*}$ \\
\hline Yan et al. [8] & 0.410 & 0.871 & 0.780 & $0.439 *$ & $0.424 *$ \\
\hline BindN (Wang and Brown) [40] & 0.652 & 0.728 & 0.722 & $0.186 *$ & $0.289 *$ \\
\hline DP-Bind (Hwang et al.) [7] & 0.791 & 0.786 & 0.800 & $-*$ & $-*$ \\
\hline
\end{tabular}

*The numbers with an asterisk are those that have been derived from the numbers reported in the related studies.

sequence-specific binding corresponds to sequencespecific recognition of a gene and is therefore essential for correct gene regulation. However, non-specific binding residues can help specific binding residues to increase binding specificity as well.

In the experiments reported in this article, our proposed approach has been able to deliver precision $81.70 \%$ and $65.47 \%$ in sequence-specific and non-specific binding residue prediction respectively. Precision of $81.70 \%$ implies that about 4 out of 5 predicted binding residues are really involved in sequence-specific binding with the DNA. Precision of $65.47 \%$ implies that about 7 out of 10 predicted binding residues are really involved in nonspecific binding with the DNA. While combining prediction results, the performance for DNA-binding residue prediction can deliver sensitivity $56.85 \%$. Sensitivity of $56.85 \%$ implies that our proposed approach can catch about 6 out of 10 residues involved in DNA binding with the DNA. In the DNA-binding segment of the protein, regions where non-specific binding residues are located will cover the regions where specific binding residues are located. Therefore, improvement can be achieved for DNA-binding residues prediction while combining prediction results of specific and non-specific binding residues. The protein-DNA binding mode prediction is also proposed in this framework, and we select 1LMB:4 as an example to reveal how can be helpful for improving DNA-binding residue prediction.

It is anticipated that the prediction accuracy delivered by our proposed approach will continue to improve as the number of TF-DNA complexes deposited in the PDB continues to grow which will increase the number of training samples for use in our learning algorithm. Nevertheless, the primary interest of computational biologists is to develop more advanced prediction mechanisms. In this respect, we believe that as the number of TF-DNA complexes deposited in the PDB increases, we can obtain more insights about the key physiochemical properties that play essential roles in TFDNA interactions to be used to develop more advanced prediction mechanisms. In addition, we will exploit the experiences learned in this study in order to design binding-mechanism concerned predictors for other families of proteins interacting with DNA. We believe that different families of proteins may have very different characteristics. Therefore, a specifically-designed predictor should be created for each specific type of protein to be able to deliver superior performance in comparison with a general-purpose predictor.

\section{Materials and methods \\ Datasets}

Our analysis was based on the dataset of DNA-binding residue prediction collected by Ofran and Rost [6]. In this collection, there are 691 protein-DNA complexes. Because we focus on transcription factors, we have created a data set containing 253 TF-DNA complexes among which 227 complexes were extracted from the 691 protein-DNA complexes, and the remaining 26 TFDNA complexes are those that were deposited into PDB between September 2007 and November 2008. All protein structures are determined by X-ray crystallization at a resolution of $3.5 \AA$ or better. Using the Gene Ontology (GO) terms [41], we use proteins where the molecular function is transcription factor activity, biological process is transcription, and cellular component is nucleus to select transcription factors. All $253 \mathrm{TF}-$ DNA complexes are listed in Table 5.

\section{Defining the DNA-binding residue}

Previous research used various distance cut-offs from $3.5 \AA$ to $6 \AA$ to define DNA-binding residues between proteins and DNA [6-10,14,40,42]. Most, if not all, of the cut-off distance is measured between the atoms of amino acid and the atoms of nucleotide bases or sugarphosphate backbones. Most DNA-binding residue prediction tools used $3.5 \AA$ or $4.5 \AA$ as the distance cut-off in general. Considering electrostatic interaction, hydrogen bonding, water-mediated hydrogen bonding, and van der Waals contacts, we use $4.5 \AA$ distance cut-off to label DNA-binding residues. A residue is regarded as involved 
Table 5: Dataset of 253 TF-DNA complexes for DNA-binding residues prediction

\begin{tabular}{|c|c|c|c|c|c|c|c|c|c|}
\hline \multicolumn{10}{|c|}{253 TF-DNA Complexes } \\
\hline $\mathrm{IA02:F}$ & IA02:J & IAOA:A & $\mathrm{IAOA}: \mathrm{B}$ & IA6Y:A & IA6Y:B & IAKH:A & $\mathrm{IAKH}: \mathrm{B}$ & IAM9:A & IAM9:B \\
\hline IAM9:C & IAM9:D & IAN2:A & IAN4:A & IAN4:B & IAPL:C & IAPL:D & IAU7:A & IAU7:B & $|B 0|: A$ \\
\hline$|\mathrm{BO}|: \mathrm{B}$ & IB72:B & IB8I:B & IBDT:A & IBDT:B & IBDT:C & IBDT:D & IBDV:A & IBDV:B & IBDV:C \\
\hline IBDV:D & IBY4:A & IBY4:B & IBY4:C & IBY4:D & ICOW:A & ICOW:B & ICOW:C & ICOW:D & ICF7:A \\
\hline ICF7:B & ICGP:A & ICGP:B & ICMA:A & ICMA:B & ICQT:A & ID5Y:A & ID5Y:B & ID5Y:C & ID5Y:D \\
\hline ID66:A & ID66:B & IDDN:A & IDDN:B & IDDN:C & IDDN:D & IDSZ:A & IDSZ:B & IDUO:A & IDUO:B \\
\hline IEA4:A & IEA4:B & IEA4:D & IEA4:E & IEA4:F & IEA4:G & IEA4:H & IEA4:J & IEA4:K & IEA4:L \\
\hline |F2|:G & $|F 2|: H$ & |F2|:I & IF2I:J & IF2I:K & |F2|:L & IF5T:A & IF5T:B & IF5T:C & IF5T:D \\
\hline IFJL:A & IFJL:B & IFJL:C & IFOS:E & IFOS:F & IFOS:G & IFOS:H & IG2D:C & IG2D:F & IG2F:C \\
\hline IG2F:F & IGDT:A & IGDT:B & IH88:A & IH88:B & IH89:A & IH89:B & IH8A:A & IH8A:B & IH9T:A \\
\hline IH9T:B & IHCQ:A & IHCQ:B & IHDD:C & IHDD:D & IHFO:A & IHFO:B & IHJB:A & IHJB:B & IHJB:D \\
\hline IHJB:E & IHLO:A & IHLO:B & IHW2:A & IHW2:B & IHWT:C & IHWT:D & IHWT:G & IHWT:H & IIO4:A \\
\hline IIO4:B & IJGG:A & IJGG:B & IJNM:A & IJNM:B & IJTO:A & IJTO:B & IJTO:C & IJTO:D & IJWL:A \\
\hline IJWL:B & |K6|:A & $|\mathrm{K} 6|: B$ & IK6I:C & $|K 6|: D$ & IKB2:A & IKB2:B & IKB4:A & IKB4:B & IKB6:A \\
\hline IKB6:B & IKU7:A & IL3L:A & IL3L:B & IL3L:C & IL3L:D & ILAT:A & ILAT:B & ILB2:A & ILE8:A \\
\hline ILE8:B & ILLI:A & ILLI:B & ILLM:C & ILMB:3 & ILMB:4 & IMDY:A & IMDY:C & IMDY:D & IMEY:C \\
\hline IMEY:F & IMJM:A & IMJM:B & IMJP:A & IMJP:B & IMNM:C & IMNM:D & INKP:A & INKP:B & INKP:D \\
\hline INKP:E & INLW:A & INLW:B & INLW:D & INLW:E & IP47:A & IP47:B & IPAR:A & IPAR:B & IPAR:C \\
\hline IPAR:D & IPER:L & IPER:R & IPUF:A & IPUF:B & IPYI:A & IPYI:B & IQP9:A & IQP9:B & IQP9:C \\
\hline IQP9:D & IRON:A & IRPE:L & IRPE:R & ITF6:A & ITF6:D & ITRO:A & ITRO:C & ITRO:E & ITRO:G \\
\hline ITRR:A & ITRR:B & ITRR:D & ITRR:E & ITRR:G & ITRR:H & ITRR:J & ITRR:K & IYRN:A & IYRN:B \\
\hline IYSA:C & IYSA:D & IZME:C & IZME:D & 2DRP:A & 2DRP:D & 2HAP:C & 2HAP:D & 2HDD:A & 2HDD:B \\
\hline 2NLL:A & 2NLL:B & 2ORI:L & 2ORI:R & 2PRT:A & 2QL2:A & 2QL2:B & 2QL2:C & 2QL2:D & 2R5Y:A \\
\hline 2R5Y:B & 3BPY:A & 3CBB:A & $3 \mathrm{CBB}: \mathrm{B}$ & 3CO6:C & 3COQ:A & 3COQ:B & 3D0A:A & 3D0A:B & 3D0A:C \\
\hline 3D0A:D & 3DFX:A & 3DFX:B & 3DZY:A & 3DZY:D & $3 \mathrm{E} 00: \mathrm{A}$ & $3 E 00: D$ & 3EXJ:A & $3 \mathrm{EXJ}: \mathrm{B}$ & 3EXL:A \\
\hline 3HDD:A & 3HDD:B & 9ANT:A & & & & & & & \\
\hline
\end{tabular}

in sequence-specific binding with DNA if one or more heavy atoms on its side-chain are within $4.5 \AA$ from the nucleic bases of the DNA. A residue is regarded as involved in non-specific binding with the DNA, if one or more heavy atoms on its side-chain are within $4.5 \AA$ from the sugar/phosphate backbone of the DNA. In all 253 TF-DNA complexes, there are 1526 binding residues and 23371 non-binding resides for sequence-specific binding residue prediction. The ratio of positive to negative samples is $1: 15$ in sequence-specific binding. For non-specific binding residue prediction, there are 3831 binding residues and 21066 non-binding residues. The ratio of positive to negative samples is $1: 5$ in nonspecific binding. The number of non-specific binding residues is twice as many as the number of sequencespecific binding residues. Without distinguishing between sequence-specific and non-specific binding residues, there are 4360 binding residues and 20537 non-binding residues. All missing residues which do not have coordinate information in the PDB data file, will be excluded from the training and testing datasets.

\section{Framework of DNA-binding residues and binding mode prediction using support vector machine}

We proposed the two stage framework to predict the DNA-binding residues in a protein and the corresponding binding mode for a query protein respectively. Figure 3 shows the overall framework for binding residue prediction and a binding mode prediction. The first stage predicts the DNA binding residues and the second stage predicts the protein-DNA binding mode. In the first stage, a well-known machine leaning approach has been used for prediction from amino acid sequences which uses support vector machine with features created by the evolutionary profile of the proteins $[43,44]$. The evolutionary profile of position-specific scoring matrices (PSSM) is computed by PSI-BLAST [45] against the NR

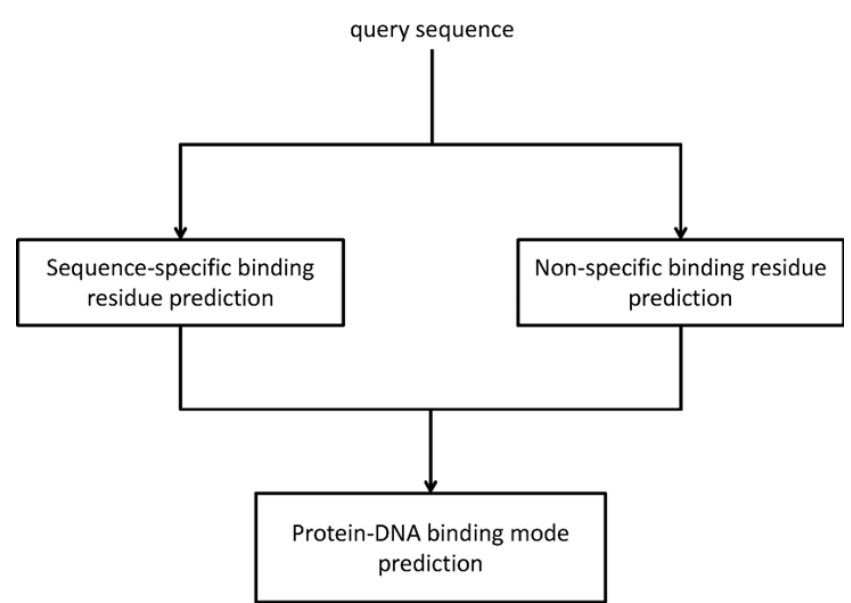

Figure 3

Overall framework for DNA-binding residues prediction. 
database for a protein sequence. In addition, in order to keep evolutionary information of neighborhood residues information, we use the principle of sliding window to calculate the backward (or/and forward) metrics over a limited region of the received sequence. For each residue in a protein sequence, we use a sliding window of size 11 to describe neighborhood information; therefore, we have a $11 * 21=231$ dimension feature factor in addition to the 20 amino acids and a boundary flag. In the end, we used LIBSVM [39] as predictor to predict DNA-binding residues. The best parameters selected for DNA-binding residues prediction is decided by leave-one-out cross validation (LOOCV).

In second stage, protein-DNA binding mode is predicted by using the prediction results of the previous stage. In Table 6, DNA-binding domains recognized by Pfam [46] will be classified into five binding modes, including zipper-type, helix-turn-helix (HTH), zinc-coordinating, $\beta$-hairpin/ribbon, and others. As shown in Table 7 , there

Table 6: Protein-DNA binding modes and their corresponding Pfam domains

\begin{tabular}{|c|c|}
\hline Protein-DNA Binding mode & Pfam Domain \\
\hline Zipper-type & $\begin{array}{l}\text { HLH } \\
\text { bZIP_I } \\
\text { bZIP_2 }\end{array}$ \\
\hline Helix-turn-helix & $\begin{array}{l}\text { HTH_AraC } \\
\text { TetR_N } \\
\text { Trp_repressor } \\
\text { Homeobox } \\
\text { E2F_TDP } \\
\text { GntR } \\
\text { Fe_dep_repress } \\
\text { Ets } \\
\text { HTH_3 } \\
\text { Sigma70_r4 } \\
\text { Lacl } \\
\text { Fork_head } \\
\text { Resolvase } \\
\text { Pou }\end{array}$ \\
\hline Zinc-coordinating & $\begin{array}{l}\text { zf-C4 } \\
\text { zf-C2H2 } \\
\text { Zn_clus } \\
\text { GATA } \\
\text { P53 }\end{array}$ \\
\hline$\beta$-hairpin/ribbon & $\begin{array}{l}\text { Met] } \\
\text { RHH_I } \\
\text { Arc }\end{array}$ \\
\hline Others & $\begin{array}{l}\text { SRF-TF } \\
\text { Runt } \\
\text { PAX } \\
\text { RHD } \\
\text { Autoind_bind } \\
\text { cNMP_binding }\end{array}$ \\
\hline
\end{tabular}

Table 7: Illustration of feature set for protein-DNA binding mode prediction

\begin{tabular}{ll}
\hline Feature title & Feature descriptions \\
\hline Class label & 5 protein-DNA binding modes \\
& I. zipper-type \\
& 2. helix-turn-helix (HTH) \\
& $\begin{array}{l}\text { 3. zinc-coordinating } \\
\text { 4. } \beta \text {-hairpin/ribbon } \\
5 . \text { others }\end{array}$ \\
& 20 dimensions of amino acid \\
Non-specific binding & 3 dimensions of secondary structure \\
& elements \\
& $\#$ of binding residues \\
\hline Protein chain information & 3 dimensions of secondary structure \\
& elements \\
& \# of total residues in a protein chain \\
\hline
\end{tabular}

are 28 features for protein-DNA binding mode prediction including the information of non-specific binding residues, predicted secondary structure elements, and the number of total residues. The secondary structure elements for each protein structure in the training data are determined by DSSP program [47]. Because this predictor is a sequence based predictor to identify protein-DNA binding mode, the secondary structure elements for each protein structure in testing data (query protein) are predicted by PSIPRED [48]. In the training dataset, we used only the residue information in DNAbinding domain detected by Pfam server.

\section{Predictor performance measures}

The predictions made for the testing instances are compared with the defined class labels (binding or non-binding) to evaluate the predictor. The accuracy is defined as

$$
\text { Accuracy }=\frac{T P+T N}{T P+T N+F P+F N}
$$

where TP is the number of true positives (binding residues with positive predictions); TN is the number of true negatives (non-binding residues with negative predictions); FP is the number of false positives (nonbinding residues but predicted as binding sites) and FN is the number of false negatives (binding residues but predicted as non-binding sites). Since the data for DNAbinding residue prediction is skewed, the accuracy alone may be misleading. The predictor can achieve 85\% accuracy by simply predicting all residues as negative for datasets where the positive to negative sample ratio is $1: 10$. Therefore, we focus on the specificity and sensitivity of the predictions, which are defined as follows: 


$$
\begin{aligned}
& \text { Sensitivity }=\frac{T P}{T P+F N} \\
& \text { Specificity }=\frac{T N}{T N+F P}
\end{aligned}
$$

The sensitivity is used to measure the prediction capability of positive samples; the specificity is used to measure the prediction capability of negative samples. In addition, precision and F-measure are also defined as follows:

$$
\begin{gathered}
\text { precision }=\frac{T P}{T P+F P} \\
F-\text { measure }=\frac{2 \times \text { precision } \times \text { Sensitivity }}{\text { precision } \times \text { Sensitivity }}
\end{gathered}
$$

\section{List of abbreviations used}

GO: Gene Ontology; LOOCV: Leave-one-out crossvalidation; NDB: Nucleic Acid Database; PDB: Protein Data Bank; SVM: Support vector machine; TFs: Transcription factors;

\section{Competing interests}

The authors declare that they have no competing interests.

\section{Authors' contributions}

YFH and CCH developed and implemented the overall framework and drafted the manuscript; YCL provided valuable suggestion on dealing with dataset and proteinDNA complexes. YFH and CKH edited the manuscript and introduced the problem initially. YJO gave suggestions on fundamental knowledge of machine learning. All authors have read and approved the final manuscript.

\section{Note}

Other papers from the meeting have been published as part of BMC Bioinformatics Volume 10 Supplement 15, 2009: Eighth International Conference on Bioinformatics (InCoB2009): Bioinformatics, available online at http://www.biomedcentral.com/1471-2105/10? issue $=\mathrm{S} 15$.

\section{Acknowledgements}

This research has been supported by the National Science Council and National Taiwan University. Funding for open access charge: National Science Council, NSC 97-2627-P-00I-002.

This article has been published as part of BMC Genomics Volume 10 Supplement 3, 2009: Eighth International Conference on Bioinformatics (InCoB2009): Computational Biology. The full contents of the supplement are available online at http://www.biomedcentral.com//47/-2/64/I0? issue $=\mathrm{S} 3$.

\section{References}

I. Berman HM, Westbrook J, Feng Z, Gilliland G, Bhat TN, Weissig H, Shindyalov IN and Bourne PE: The Protein Data Bank. Nucleic Acids Res 2000, 28(I):235-242.

2. Berman HM, Olson WK, Beveridge DL, Westbrook J, Gelbin A, Demeny T, Hsieh SH, Srinivasan AR and Schneider B: The nucleic acid database. A comprehensive relational database of three-dimensional structures of nucleic acids. Biophys J I992, 63(3):75I-759.

3. Reddy CK, Das A and Jayaram B: Do water molecules mediate protein-DNA recognition? J Mol Biol 200I, 3|4(3):6|9-632.

4. Luscombe NM and Thornton JM: Protein-DNA interactions: amino acid conservation and the effects of mutations on binding specificity. J Mol Biol 2002, 320(5):99|-1009.

5. Luscombe NM, Laskowski RA and Thornton JM: Amino acid-base interactions: a three-dimensional analysis of protein-DNA interactions at an atomic level. Nucleic Acids Res 200I, 29(I3): 2860-2874.

6. Ofran Y, Mysore $\mathrm{V}$ and Rost B: Prediction of DNA-binding residues from sequence. Bioinformatics 2007, 23(13):i347-353.

7. Hwang S, Gou Z and Kuznetsov IB: DP-Bind: a web server for sequence-based prediction of DNA-binding residues in DNA-binding proteins. Bioinformatics 2007, 23(5):634-636.

8. Yan C, Terribilini M, Wu F, Jernigan RL, Dobbs D and Honavar V: Predicting DNA-binding sites of proteins from amino acid sequence. BMC Bioinformatics 2006, 7:262.

9. Wang $L$ and Brown SJ: Prediction of DNA-binding residues from sequence features. J Bioinform Comput Biol 2006, 4(6): $1|4|-1158$

10. Ahmad S and Sarai A: PSSM-based prediction of DNA binding sites in proteins. BMC Bioinformatics 2005, 6:33.

II. Wu J, Liu H, Duan X, Ding Y, Wu H, Bai $Y$ and Sun X: Prediction of DNA-binding residues in proteins from amino acid sequences using a random forest model with a hybrid feature. Bioinformatics 2009, 25(I):30-35.

12. Bhardwaj N, Langlois R, Zhao $G$ and Lu H: Structure Based Prediction of Binding Residues on DNA-binding Proteins. Conf Proc IEEE Eng Med Biol Soc 2005, 3:26II-26I4.

13. Tsuchiya $Y$, Kinoshita $\mathrm{K}$ and Nakamura $\mathrm{H}$ : Structure-based prediction of DNA-binding sites on proteins using the empirical preference of electrostatic potential and the shape of molecular surfaces. Proteins 2004, 55(4):885-894.

14. Ahmad S, Gromiha MM and Sarai A: Analysis and prediction of DNA-binding proteins and their binding residues based on composition, sequence and structural information. Bioinformatics 2004, 20(4):477-486.

15. Jones S, Shanahan HP, Berman HM and Thornton JM: Using electrostatic potentials to predict DNA-binding sites on DNA-binding proteins. Nucleic Acids Res 2003, 3 I(24): 7189-7198.

16. Bhardwaj $\mathrm{N}$ and Lu H: Residue-level prediction of DNA-binding sites and its application on DNA-binding protein predictions. FEBS Lett 2007, 58 I(5): 1058-1066.

17. Chen YC, Wu CY and Lim C: Predicting DNA-binding amino acid residues from electrostatic stabilization upon mutation to Asp/Glu and evolutionary conservation. Proteins 2007, 67(3):67|-680.

18. Chu WY, Huang YF, Huang CC, Cheng YS, Huang CK and Oyang YJ: ProteDNA: a sequence-based predictor of sequence-specific DNA-binding residues in transcription factors. Nucleic Acids Res 2009, Web Server:, to appear.

19. Calkhoven $C F$ and $A b$ G: Multiple steps in the regulation of transcription-factor level and activity. Biochem J I996, 3 I 7(Pt 2):329-342.

20. Pabo $C O$ and Sauer RT: Transcription factors: structural families and principles of DNA recognition. Annu Rev Biochem 1992, 61:1053-1095.

21. Latchman DS: Transcription factors: an overview. Int J Biochem Cell Biol 1997, 29(12): 1305-1312.

22. Latchman DS: Transcription factors: an overview. Int J Exp Pathol 1993, 74(5):417-422.

23. Liu J, Perumal NB, Oldfield CJ, Su EW, Uversky VN and Dunker AK: Intrinsic disorder in transcription factors. Biochemistry 2006, 45(22):6873-6888

24. Kalodimos CG Biris $N$, Bonvin AM, Levandoski MM Guennuegues $M$, Boelens $R$ and Kaptein R: Structure and flexibility adaptation in nonspecific and specific proteinDNA complexes. Science 2004, 305(5682):386-389.

25. McGhee JD and von Hippel PH: Theoretical aspects of DNAprotein interactions: co-operative and non-co-operative 
binding of large ligands to a one-dimensional homogeneous lattice. J Mol Biol I974, 86(2):469-489.

26. Von Hippel PH and McGhee JD: DNA-protein interactions. Annu Rev Biochem 1972, 4I(I0):23 I-300.

27. von Hippel PH: Protein-DNA recognition: new perspectives and underlying themes. Science 1994, 263(5 I 48):769-770.

28. Boyer RF: Concepts in Biochemistry: Structure Tutorials. Concepts in Biochemistry Wiley; 32005, 736.

29. Cheng AC, Chen WW, Fuhrmann CN and Frankel AD: Recognition of nucleic acid bases and base-pairs by hydrogen bonding to amino acid side-chains. J Mol Biol 2003, 327 (4):78I-796.

30. Luscombe NM, Austin SE, Berman HM and Thornton JM: An overview of the structures of protein-DNA complexes. Genome Biol 2000, I(I):REVIEWSOOI.

31. Harrison SC: A structural taxonomy of DNA-binding domains. Nature 1991, 353(6346):715-719.

32. Luisi B: DNA-protein interaction at high resolution. DNAProtein Structural Interactions. New York: Oxford University Press: Lilley DMJ 1995, I-48.

33. Prabakaran P, Siebers JG, Ahmad S, Gromiha MM, Singarayan MG and Sarai $A$ : Classification of protein-DNA complexes based on structural descriptors. Structure 2006, I 4(9): |355-|367.

34. Wintjens R and Rooman M: Structural classification of $\mathbf{H T H}$ DNA-binding domains and protein-DNA interaction modes. | Mol Biol 1996, 262(2):294-3|3.

35. Yaginuma T: [Pressure-volume relationship. Aortic compliance - with special reference to an observation in various stress conditions]. Nippon Rinsho 1975, 33(7):2295-2303.

36. Vazquez ME, Caamano AM and Mascarenas JL: From transcription factors to designed sequence-specific DNA-binding peptides. Chem Soc Rev 2003, 32(6):338-349.

37. Siggers TW, Silkov A and Honig B: Structural alignment of protein-DNA interfaces: insights into the determinants of binding specificity. J Mol Biol 2005, 345(5): I027-1045.

38. von Hippel PH and Berg OG: On the specificity of DNA-protein interactions. Proc Natl Acad Sci USA 1986, 83(6):|608-16|2.

39. Chang C-C and Lin C-J: LIBSVM: a library for support vector machines. 200I http://www.csie.ntu.edu.tw/ cjlin/libsvm.

40. Wang $L$ and Brown SJ: BindN: a web-based tool for efficient prediction of DNA and RNA binding sites in amino acid sequences. Nucleic Acids Res 2006, 34 Web Server: W243-248.

4I. Ashburner M, Ball CA, Blake JA, Botstein D, Butler H, Cherry JM, Davis AP, Dolinski K, Dwight SS and Eppig JT, et al: Gene ontology: tool for the unification of biology. The Gene Ontology Consortium. Nat Genet 2000, 25(I):25-29.

42. Tjong $H$ and Zhou HX: DISPLAR: an accurate method for predicting DNA-binding sites on protein surfaces. Nucleic Acids Res 2007, 35(5): |465-|477.

43. Larranaga P, Calvo B, Santana R, Bielza C, Galdiano J, Inza I, Lozano JA, Armananzas R, Santafe G and Perez A, et al: Machine learning in bioinformatics. Brief Bioinform 2006, 7(I):86-II2.

44. Bhaskar $H$, Hoyle $D C$ and Singh S: Machine learning in bioinformatics: a brief survey and recommendations for practitioners. Comput Biol Med 2006, 36(I0): I I04-II 25.

45. Altschul SF, Madden TL, Schaffer AA, Zhang J, Zhang Z, Miller W and Lipman DJ: Gapped BLAST and PSI-BLAST: a new generation of protein database search programs. Nucleic Acids Res 1997, 25(I7):3389-3402.

46. Finn RD, Tate J, Mistry J, Coggill PC, Sammut SJ, Hotz HR, Ceric G Forslund K, Eddy SR and Sonnhammer EL, et al: The Pfam protein families database. Nucleic Acids Res 2008, 36 Database: D28I-288.

47. Kabsch $\mathrm{W}$ and Sander C: Dictionary of protein secondary structure: pattern recognition of hydrogen-bonded and geometrical features. Biopolymers 1983, 22(I 2):2577-2637.

48. McGuffin LJ, Bryson K and Jones DT: The PSIPRED protein structure prediction server. Bioinformatics 2000, I6(4):404-405.
Publish with Biomed Central and every scientist can read your work free of charge

"BioMed Central will be the most significant development for disseminating the results of biomedical research in our lifetime. "

Sir Paul Nurse, Cancer Research UK

Your research papers will be:

- available free of charge to the entire biomedical community

- peer reviewed and published immediately upon acceptance

- cited in PubMed and archived on PubMed Central

- yours - you keep the copyright
BioMedcentral 